\title{
A Bullet holes detection algorithm based on wavelet transform and image fusion
}

\author{
Luo Jie*, Zhang Zhiming, Zeng Guangdong \\ Information engineering department Engineering College of CAPF Xi'an, China \\ 1030915907@qq.com
}

KeyWords: object detection; wavelet transform; image fusion; image denoising; fusion rule

\begin{abstract}
A bullet holes object detection based on wavelet transform using fusion rules is proposed to overcome the limitation of the bullet holes detection using image difference technology that exists over-detection or leak-detection because of the noise interference and has harsh request for imaging condition. In the wavelet domain, it applied different fusion rules to the low-frequency and the high-frequency sub-image to revise the wavelet transform coefficients in order to increase the object coefficients while decrease or reset the non-object coefficients. Then, it used the maximum points to the low-frequency coefficient and reduced the noise of high-frequency coefficients, the final bullet holes images were obtained through wavelet composition. Experimental results show the proposed method can detect bullet holes object not only remove the noise effect but also enhance the edge, which improves the veracity and precision of the bullet holes detection.
\end{abstract}

\section{Introduction}

Target detection is the main contents of the computer vision and an important part of the image analysis and recognition [1,2]. Classic methods of target detection include inter-difference method [3] and background subtraction method [4]. Traditional bullet holes detection methods based on subtraction algorithm have high requirement of the image and imaging condition of bullet holes, prone to false alarms or missed, and the edge of the bullet holes in target detection was fuzzy and discontinuous, seriously affecting the recognition precision and accuracy.

Therefore, this paper proposes a bullet holes detection algorithm based on wavelet transform and image fusion technology. Reconstruct image using multi-scale edge of the image according to multi-scale edge image fusion proposed by Nikolov [5,6]. When carrying on the wavelet inverse transform, we cannot merge all the wavelet coefficients on each frequency[7]. Before detection, carrying on n-layer wavelet transform, the image is decomposed into different frequency regions by adopting different methods in different frequency after processing. Then increasing the Low and High wavelet transform frequency coefficient of target area[8], and reducing or setting zero to the non-target area wavelet coefficients, so the fusion image contains only the target object after wavelet inverse transform, resulting in continuous edge, clear target image which increases the precision of target location and suppresses noise.

\section{Related Works}

Multi-Scale Edge Processing Based on Wavelet Transform (Maxima Modulus Method). We

supposed a two-dimensional smoothing function satisfies as: $\theta(u, v) \geq 0, \iint_{R^{2}} \theta(u, v) d u d v=1, \lim _{u, v \rightarrow \pm \infty} \theta(u, v)=0$

Remember $\theta_{2^{j}}(u, v)=\frac{1}{2^{2 j}} \theta\left(\frac{u}{2^{j}}, \frac{v}{2^{j}}\right)$. Then, the mold for gradient vector is $\operatorname{Mf}\left(2^{j}, u, v\right)=\sqrt{\mid W^{1} f\left(2^{j}, u,\left.v\right|^{2}+\mid W^{2} f\left(2^{j}, u,\left.v\right|^{2}\right.\right.}$

And the angle of Gradient direction and the horizontal (phase angle or amplitude angle) is 


$$
\operatorname{Af}\left(2^{j}, u, v\right)=\operatorname{arctar}\left(\left(w^{2} f\left(2^{j}, u, v\right)\right) /\left(w^{1} f\left(2^{j}, u, v\right)\right)\right)
$$

using formula (1), (2) to calculate the points with local maximum modulus of sub image wavelet transform, which are the image edge points on the scales of $2^{j}$, and the corresponding wavelet coefficients of the edge is obtained.

Denoising Algorithm Based on Wavelet Transform. After wavelet transform, noise and image details are respectively concentrated in smaller amplitude wavelet coefficients and large amplitude wavelet coefficients of the high frequency region. According to this feature, this paper uses an adaptive de-noising algorithm for high-frequency region. Different coefficients are multiplied by the noise reduction factor which is less than or equal 1, associated with itself absolute value of the wavelet coefficients, and the factor exponentially decreases with the increase of the wavelet coefficients. The algorithm is able to cater for different wavelet decomposition levels, so that we can retain some useful detail information while remove image noise.

\section{Bullet Holes Target Detection Algorithm}

The main purpose of target detection is to highlight target object and target edge details. Wavelet transform on different scales provides a certain amount of edge information of the original image. When applying this feature to bullet holes detection, under large-scale, we determine the rough edge of the point of impact area, and in small scale, further precisely pinpoint bullet holes' edges.

After wavelet decomposition, target edge is often mixed together with a lot of noise points, especially the high frequency region. Therefore, edges details are blur or discontinuous. To solve this problem, we propose to adopt the modulus evolution to low frequency and wavelet noise reduction to high frequency areas before wavelet inverse transformation.

Based on the above theory, the basic idea of bullet holes target detection is as follows: First, through $\mathrm{N}$ layer wavelet decomposition, image is divided into $3 \mathrm{~N}$ high frequency bands, which contains the image details and a low frequency band containing global information; then, we respectively take rough and precise detection to the low-frequency and high-frequency portion of the target area. According to the results of above detection, we increase the wavelet transform coefficients of the object region, while reduce or set 0 to the non-target transform coefficients. Then we process modulus maxima to the wavelet coefficients of the low-frequency sub-images, and use wavelet noise reduction to wavelet coefficients of high-frequency sub-images. Finally, by taking the wavelet inverse transform to sub-images, we can obtain detected image containing only bullet holes. Figure 1 shows the flow chart of object detection algorithm

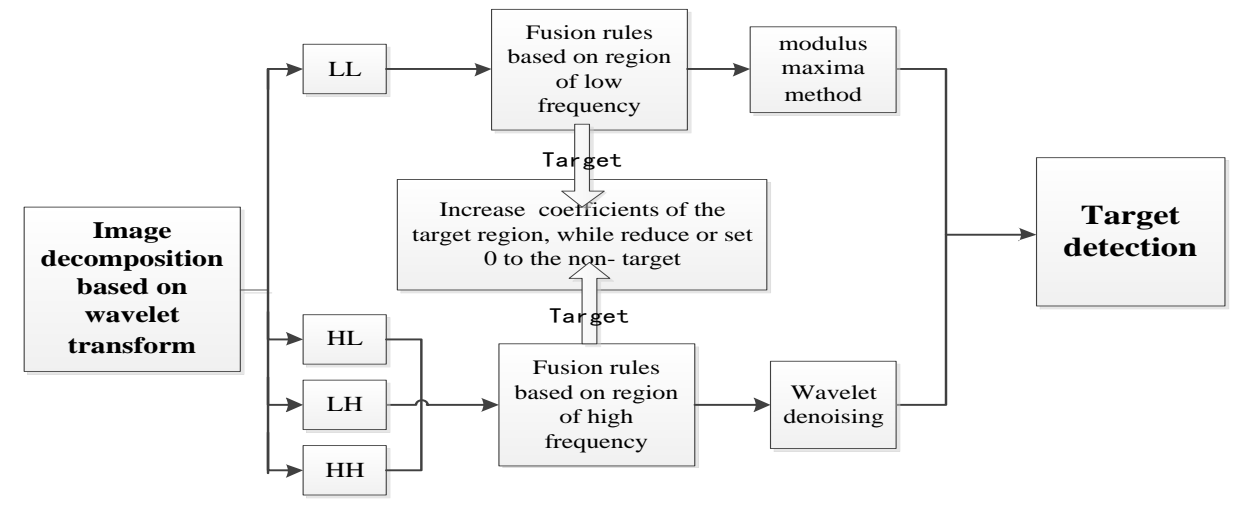

Fig. 1.Flow chart of object detection algorithm

Specific ideas of algorithm are as follows: 
- Take wavelet decomposition to both of image with no-bullet targets and bullet holes targets, and establish the corresponding data of wavelet decomposition.

- In each layer of wavelet decomposition, take regional integration to wavelet decomposition coefficients of different frequency bands, which at the same region of two images .fusion rules use the local field method, and window size of field is $3 \times 3$, with the mean of field as the characteristic values, to improve the wavelet transform coefficients of area containing bullet holes targets so as to highlight the edge and position of the bullet target. Specific fusion rules are divided into low frequency fusion rules and the high frequency part of the fusion rules.

Fusion rules of Low frequency part: Since the low-frequency portion contains the global information of image, it is the basis for the reconstruction of the wavelet transform. Therefore, for this part of the main objectives is to highlight the continuity of bullet holes' edge. for Low frequency part, we calculate the mean of local field and the global field of the two images' wavelet transform coefficients, if the absolute value of the difference between the mean is less than or equal to the global average in corresponding field, indicating that there is no target information in the field, we set the corresponding coefficients of image containing bullet hole targets to zero. Otherwise, the current coefficient represents the coefficient of bullet target area. Then take the maximum value of the field to modify the corresponding coefficients of image containing bullet targets

Fusion rules of High-frequency part: first of all, take the corresponding low frequency information into consideration, if low-frequency coefficient of 0 ,which indicates a pseudo-edge, set the corresponding high-frequency coefficients of image containing bullet hole targets to zero; otherwise, respectively acquire the local mean of different high-frequency coefficients of image without target and the corresponding high-frequency coefficients of center of the field of image with bullet target In the corresponding field: in each of the corresponding high frequency, if the high-frequency coefficient is greater than a threshold value while the local filed mean of high-frequency coefficient is smaller than the threshold, which means that the coefficient represents the target transform coefficients and remains unchanged; otherwise set the coefficients to zero.

- For the low-frequency coefficients of the first layer to the $\mathrm{N}$ layer, use modulus maxima method, to get the low-frequency coefficients of target edge wavelet transform.

- For high frequency coefficient, using the above-mentioned wavelet de-noising algorithm to deal with the noise.

- Take the inverse wavelet transform to image existence of bullet holes target to reconstruct image.

Since the amount of data to be processed is very large in the image fusion, to reduce time of bullet holes target detection and recognition and improve real-time of algorithms, the algorithm was designed to discard the former image which does not contain the target, and only take the inverse wavelet transform to image containing the target restore the image when reconstructing image, greatly improving the efficiency of the algorithm.

\section{Experimental Results and Analysis}

In order to verify the validity and accuracy of the algorithm, we conducted contrast experiment of the classical algorithm and the algorithm proposed in this paper against multiple dimensions of 256 grayscale image. In our experiment, we choose the Harr wavelet to conduct wavelet transform, and the decomposition level is 2 layers. Through the analysis of high frequency coefficients of wavelet transform, we found the most appropriate determination threshold value of high-frequency portion of the fusion rule is two. The difference of detection results between classical algorithms 
and our algorithm are shown in Figure 2:

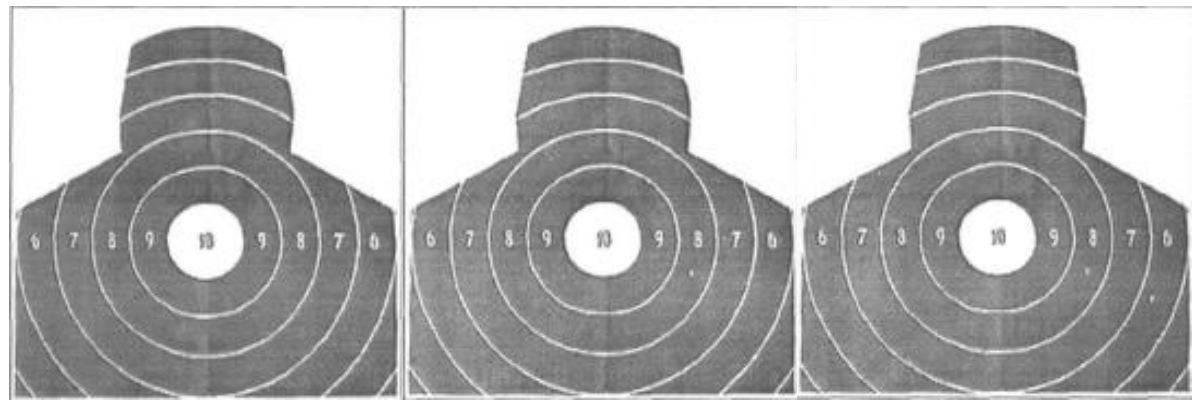

(a) image without bullet holes

image with a bullet hole

image with two bullet holes

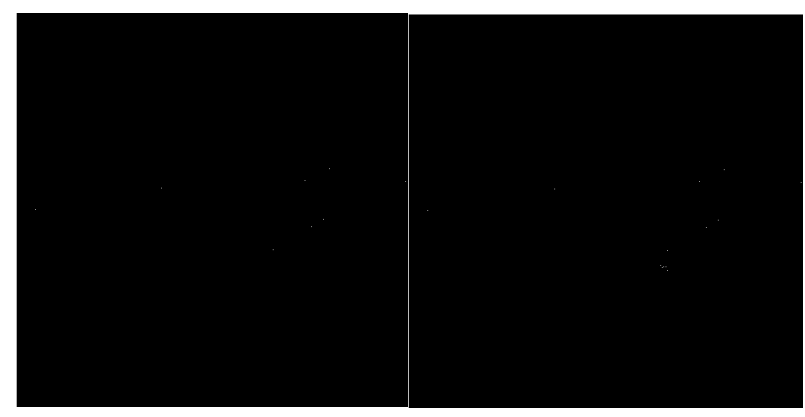

(b) detection results of single Gaussian model

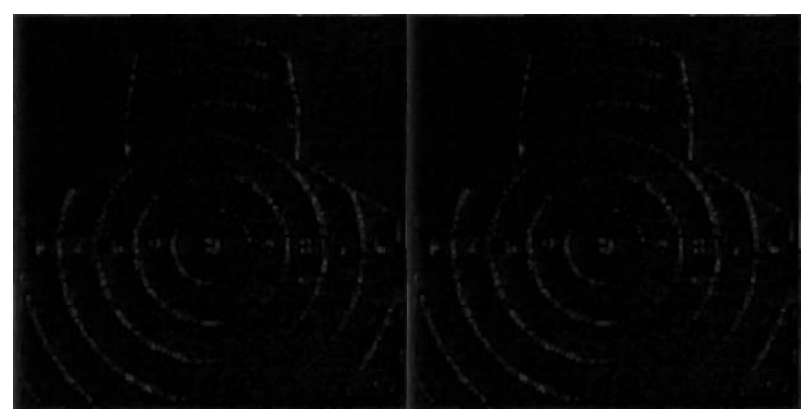

(d)detection results of subtraction method

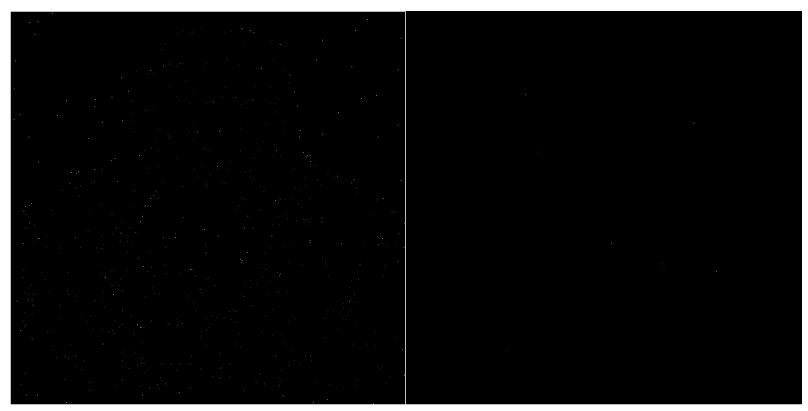

(c) detection results of Gaussian mixture model

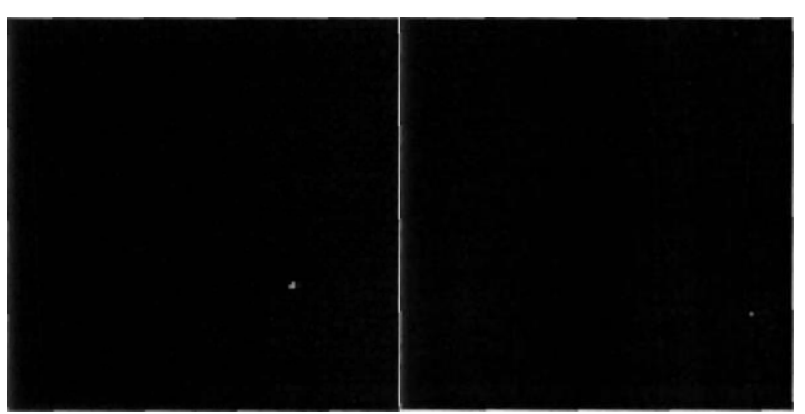

(e) detection results of algorithm in the paper

Fig. 2. Object detection image using different algorithms

It is given in Figure 2 that comparison results aimed at the case of single bullet hole and two bullet holes, using subtraction method, single Gaussian model, Gaussian mixture model and the proposed algorithm.

As can be seen from Figure 2, the subtraction method has poor suppressed effect on target contour loop; the Gaussian model method overcome the question existing in subtraction method, but the noise suppression effect is poor. There a lot of pseudo bullet holes which have similar feature with bullet holes; the algorithm of this paper can overcome the defect of the subtraction method and the Gaussian model. It successfully increases gray difference among target and background and noise to the extent to which easy to split and suppresses a lot of noise, increasing the accuracy of location identification of bullet holes. Thus, target detection algorithm based on wavelet transform and fusion technology has been greatly improved in accuracy and precision aspects.

Table 1 is the running time for the algorithm in comparison test. The data in the table shows that the running time of our algorithm is slightly higher than that of subtraction method, while lower than that of single Gaussian model and Gaussian mixture model. 
TABLE I. THE RUNING TIME OF ALGORITHMS

\begin{tabular}{lc}
\hline Algorithm & $\begin{array}{c}\text { Average } \\
\text { running time(s) }\end{array}$ \\
\hline subtraction & 0.06 \\
single Gaussian model & 2.01 \\
Gaussian mixture model & 3.41 \\
Our algorithm & 0.92 \\
\hline
\end{tabular}

TABLE II. THE BARYCENTRE COORDINATE OF EXPERIMENTAL AND REAL BULLET HOLE

\begin{tabular}{ccc}
\hline image & detected values(pixel) & measured value \\
\hline $\mathrm{a}, \mathrm{b}$ & $(183,84)$ & $(183,84)$ \\
$\mathrm{b}, \mathrm{c}$ & $(225,62)$ & $(225,64)$ \\
\hline
\end{tabular}

In order to verify the precision of the algorithm, we compared the center of gravity coordinates calculated in experiment and that of the actual measurement. As can be seen from Table 2, the biggest difference of the detected value and the actual value of the bullet holes target is between two pixels. Due to the high precision of targets' barycentre coordinate, algorithm presented in this paper can well meet the practical requirement of the system.

\section{Conclusion}

Aiming at the problem of the bullet target detection under complicated background, this paper proposed a target detection algorithm based on the wavelet transform and image fusion technology. First using wavelet transform to decompose the image into different frequency, then adopting different fusion rules and processing methods in different frequencies to increase the wavelet transform coefficient of target image, while setting the coefficient of non-target image to 0 , after wavelet inverse transformation we obtain the detection image containing bullet hole target only. The experimental results demonstrate the effectiveness and feasibility of the proposed algorithm. Comparing with subtraction detection algorithm and Gaussian model algorithm, using our algorithm to detect bullet holes can not only overcome the problems of poor imaging condition but also suppress the noise and obtain more clear, continuous edges of bullet holes, which dramatically enhanced the accuracy and precision the bullet target detection.

\section{References}

[1]. H.Z Jiang,J.J Wang, Y.H Gong. et.al. Online multi-target tracking with unified handing of complex scenarios [J].IEEE Transactions on Image Processing,2015,24(11):3464-3477.

[2]. Bo Du, L.P.Zhang. Target detection based on a dynamic subspace[J].Pattern Recognition, 2014,47:344-358.

[3]. Li Y,Z.X.SUN,Yuan B,et.al. An improved method for motion detection by frame difference and background subtraction[J]. Journal of Image and Graphics,2009,14(6):1162-1170.

[4]. Yu Y,Cao M.W,Yu F. Evibe: An improved Vibe algorithm for detecting moving objects[J].Chinese Journal of Scientific Instrument. 2014,35(4):925-932.

[5]. Gao Y, Wang A. M, Wang F. H, et.al. Application of improved wavelet transform algorithm in image fusion[J].Laser Technology. 2013,37(5):690-695.

[6]. Varshney P. k. Distributed Detection and Data Fusion[M]. New York: Springer-Verlag, 1997.

[7]. G.S.El-taweel, A.K.Helmy. Image fusion scheme based on modified dual pulse coupled neural network[J].IET Image Process,2013,7(5):407-414.

[8]. G. Bhatnagar, Q. M. Jonathan Wu, Z Liu. Directive contrast based multimodal medical image fusion in NSCT domain[J].IEEE Transactions on Multimedia,2013,15(5):1014-1024. 